\title{
Der Effekt
}

\author{
Erhard Taverna
}

Der Effekt hat einen Namen und eine Geschichte. Ein Psalm liefert den Begriff: «Placebo domino in regione vivorum.» Gesungen bei der Totenvesper als Kehr- und Rahmenvers: «Ich werde dem Herrn angenehm sein im Lande des Lebens.» Als «Placebo» wurde die Totenandacht bezeichnet, später hiess «ein Placebo singen» eine hochgestellte Persönlichkeit schmeicheln, dann wurde der Schmeichler selbst zum «Placebo». Im «Philadelphia Medical Dictionary» von 1808 wird das Wort zur «commonplace method of medicine». Ein grosser Teil der Medizingeschichte ist eine Geschichte des Placeboeffektes.

Zu Beginn meiner Praxis waren von der Apotheke unappetitliche Dragées als «Bullshitol» erhältlich. Eine moderne, substanzlose Blisterpackung in ansprechender Form hätte eigens angefertigt werden müssen und war damit viel zu teuer.

\section{Placebo}

In meiner ganzen Aus- und Weiterbildung existierte dieser Begriff nur im abwertenden Sinn, als Scheinlösung, Pseudohandlung oder Täuschung. Das wirkte noch in der Balint-Gruppe nach, wo die Kurzformel vom «Arzt als Droge» für mich einen anrüchigen Beigeschmack hatte, trotz Balints klarem Konzept einer patientenorientierten und interaktionszentrierten Medizin. Dabei hatte sich seit 1948 eine neue experimentelle Methode durchgesetzt, die Kontrollgruppen in randomisierten Studien mit einem Surrogat behandelte. Obwohl seither die «Scheinbehandlung» zum wissenschaftlichen Standard gehörte, war ihre Wirkung kein Vorlesungsthema, zu gross war die Berührungsangst vor Scharlatanen und Quacksalbern. Skrabanek und McCormick haben 1989 in ihrem Klassiker «Follies and Fallacies in Medicine» mit ätzenden Kommentaren auf Schwachstellen hingewiesen: «Faith in placebos is advantageous to both doctor and patient; faith in religion to both priest and penitent. Critical enquiry is subversion in one context and blasphemy in the other.» Wer um seine Rituale bangt, hat grundsätzliche Bedenken gegen eine evidenzbasierte Medizin mit ihren statistischen Methoden. «The best way to improve the results of any treatment is to leave out the controls. The doctor benefits, the patient benefits, only science suffers», spotteten die gleichen Autoren. Mediziner haben die Erforschung dieses hochrelevanten Phänomens an die Soziologie, die Neurowissenschaft und die Verhaltenspsychologie delegiert. Immer noch ängstigt dieser Poltergeist, der die biomedizinische Ordnung und damit unser tägliches Tun an der Nase herumführt. Doch das ändert sich langsam, denn viel versprechen die Chancen, gelänge es nur, das Potential der Selbstheilung gezielter als bisher einzusetzen. Wie Fachleute an einer Konferenz der National Institutes of Health (NIH) in Bethesda vor drei Jahren zeigten, fehlt noch viel zum Verständnis dieser eigenmächtigen Mechanismen. Doch soviel ist klar: Placebos wirken über das dopaminerge Belohnungssystem des Gehirns und verändern den Stoffwechsel in funktionellen Kernspinaufnahmen. Sie aktivieren wie Opiate entsprechende Regionen des Hirnstammes. Doch was Studien statistisch signifikant nachweisen, muss klinisch nicht immer relevant sein. Zweifellos mobilisiert die Erwartung einer Besserung die Selbstheilungskräfte. Doch diese Erwartung wird durch komplexe Interaktionen beeinflusst, und zudem wissen wir wenig über den Spontanverlauf einer Heilung. Zu Recht kommt deshalb die Cochrane Collaboration zum vorläufigen Schluss, dass die Verwendung von Placebos nur für die klinische Erforschung empfohlen werden kann. Die bewusste Anwendung in der Praxis gilt als unethisch, wenn sie ohne Einverständnis geschieht.

\section{Nocebo}

Was gefällt, kann auch missfallen. Der «Kontexteffekt», verstanden als positiver Einfluss des engagierten, emotional beteiligten und optimistischen Therapeuten, beschreibt auch die negativen Einwirkungen, die einen Noceboeffekt verursachen. Ein schlechter Ruf des Behandelnden, ungenügende Information, fehlende Empathie, Zeitmangel oder unangenehme Nebenwirkungen sind die häufigsten Ursachen. Das Paradebeispiel für diese negative Wirkung demonstriert der Beipackzettel. Die Auflistung unangenehmer und schädlicher Nebenwirkun- 
gen überspielt den Placeboeffekt. Mangelnde Motivation oder fehlendes Vertrauen des Patienten, vorgefasste Meinungen, verstärkt durch die Ängste der mitlesenden Angehörigen, führen zum Therapieabbruch oder zu falscher Dosierung. Ob positive oder negative Auswirkungen, die endokrinen und cerebralen Veränderungen unterscheiden sich nicht. Die Wege der Wahrnehmung eines gesundheitsabträglichen Effektes sind bei Versuchspersonen die gleichen wie beim Placeboexperiment. Unter besonderen Umständen sind Nocebos sogar ansteckend. Der Umgang mit vermeintlichen Risiken kann ganze
Gesellschaften krank machen und zu neuen Diagnosen führen. Ein selten erwähnter Nebeneffekt alternativer Behandlungsverfahren ist die gleichzeitige Vermittlung abstruser Körper- und Krankheitsvorstellungen. Langfristig werden dadurch funktionelle Störungen begünstigt. Die «Nützts nüüd, so schads nüüd»-Methode hat auch ihre Schattenseiten.

«The placebo response is a complex phenomenon which is still little understood», folgerten Skrabanek und McCormick. Daran wird sich, trotz intensiver Forschung, nicht so schnell etwas ändern. 